\title{
Dual Process Strategy to Increase the Usable Power for Laser-Milling
}

\author{
Fabian Nyenhuis ${ }^{*}{ }^{1}$, Andreas Michalowski ${ }^{1}$ and Johannes L'huillier ${ }^{2}$ \\ ${ }^{1}$ Robert Bosch GmbH, Bosch Research, \\ Postbox 3002 40, D-70442 Stuttgart, Germany \\ *Corresponding author's e-mail: fabian.nyenhuis@de.bosch.com \\ ${ }^{2}$ Photonik-Zentrum Kaiserslautern e.V., Kohlenhofstr. 10, 67633 Kaiserslautern, Germany \\ and Research Center OPTIMAS, University Kaiserslautern, Erwin-Schrödinger-Straße, \\ D-67663 Kaiserslautern, Germany
}

\begin{abstract}
Nowadays it is a big challenge to exploit the available average power of ultrashort-pulsed laser systems for the production of high quality structures in metals. A dual process strategy of alternating $\mathrm{GHz}$ bursts and conventional processing helps to avoid adverse effects that occur at higher average power. The combination of polishing and ablation leads to higher ablation rates. This new strategy also meets higher quality requirements in terms of surface and edge quality. Due to the spatially and temporally limited re-melting with ultrashort pulses, no strong melt formation occurs during polishing. Consequently, this approach enables a high increase of the ablation rate without lowering the precision of ultrashort pulse milling.
\end{abstract}

DOI: $10.2961 /$ jlmn.2020.03.2009

Keywords: burst ablation, surface structuring, pulsed polishing, surface quality, milling, dual process strategy, GHz bursts, bumps, edge deepening

\section{Introduction}

Surface structuring with ultrashort laser pulses is an advanced manufacturing technology due to its flexibility, outstanding precision and applicability to almost all solids. However, the high precision results in low volume removal rates, which often makes the technology unsuitable for large-scale industrial applications. The average power of the laser systems is not a limitation. Instead, the processing speed is mostly limited by thermally induced damage [1-3]. Strongly absorbing materials, such as metals, have an optimal value for the peak fluence regarding ablation efficiency, which is $\mathrm{e}^{2}$ times the threshold fluence [3]. Since higher fluences lead to a lower ablation efficiency and consequently to a worse residual heat ratio, a high average power is usually used with high repetition rates $[1,3]$.

Nevertheless, this strategy requires a critical scanning speed in order to avoid the mentioned thermal damage to the surface caused by heat accumulation of the residual heat of each individual pulse $[1,2]$. This condition currently limits the process duration, since the number of positioning jumps and the acceleration times of scanner systems increase at high speeds. In other words, the on/off ratio of the laser and consequently the average power used decreases when the scanning speed increases. Therefore, it would be better to further increase the ablation rate of each scan. In order to achieve this increase, different approaches using pulse sequences were followed. The approaches are based on two ideas: On the one hand, the splitting of energy into several pulses to be closer to the optimal fluence, which is used for relatively long delays of several nanoseconds $[4,5]$. On the other hand, short delay bursts, so-called GHz bursts, are used so that the ablation process can reuse the residual heat of the previous pulses $[6,7]$. However, the second approach seems to be unsuitable for milling applications of metals, since strong plasma shielding effects and re-deposition of matter hinder the ablation process [3-5, 8]. Additionally, for good conductors, as copper, a very high number of pulses is necessary until an improvement of the specific removal rate is achieved [9]. Since this kind of intense heat accumulation leads to strong melt formation and ejection and consequently lowers the precision, it is questionable whether a similar result can be achieved with nanosecond lasers. However, there is a third possibility to increase the ablation rate with bursts, which avoids the problems of plasma shielding, re-deposition of matter or melt ejection and therefore loss of precision. This possibility makes use of a dual process strategy, which includes a $\mathrm{GHz}$ burst polishing process [11]. The bursts are only used for polishing at relatively low fluences, so that there is no strong plasma formation or melt ejection. Applying the polishing step between ablation scans prevents surface degradation. This makes it possible to work below the critical scanning speed, which finally leads to a reduction of the process time while maintaining a high surface quality. For the example geometry we chose, a doubling of the ablation rate was achieved. On top of the time saved, the edge and surface quality of the geometry is remarkable high.

\section{Experimental setup}

The thermally induced degradation of the surface during ultrashort pulse processing is well known for many materials [1-3]. For metals, it is characterized by the occurrence and growth of bumps. Since this occurrence is related to the thermal diffusion coefficient, we chose the steel 1.4301 (X5CrNi18-10) for our experiments [1]. As a lowcost stainless steel, it is of great interest for industrial manufacturing, but due to its low thermal diffusion coefficient it tends to form bumps even at moderate scanning speeds. It is therefore a suitable candidate to investigate the dual process strategy. The laser system that was used is a modified TruMricro 2030 (Trumpf GmbH + Co. KG) with an optional 
GHz-burst-mode at $1.6 \mathrm{GHz}$. The pulse duration was set to 1 ps at a central wavelength of $1030 \mathrm{~nm}$ (Yb:YAG). A spot size $2 \omega_{0}$ of $42 \mu \mathrm{m}\left(1 / \mathrm{e}^{2}\right.$ diameter, $\left.\mathrm{M}^{2} \leq 1.3\right)$ with a typical peak intensity of $5 \times 10^{11} \mathrm{~W} / \mathrm{cm}^{2}$ was applied for the ablation process. For the polishing step, a spot size of approximately $120 \mu \mathrm{m}$ was formed by defocusing into the material by about 3 Rayleigh lengths. The line distance $d_{h}$ was set to $7 \mu \mathrm{m}$ for the ablation and $20 \mu \mathrm{m}$ for the polishing. An intelliSCAN 14 scanner system (SCANLAB GmbH) and a lens with a focal length of $163 \mathrm{~mm}$ were used for beam guidance. As already mentioned, the ablation efficiency has an optimal peak fluence at $\mathrm{e}^{2}$ times the threshold fluence $F_{t h}$. Therefore, a fluence of about 1.25 times e $\mathrm{e}^{2}$ times the threshold fluence $F_{\text {th }}$ was chosen for the experiments at a repetition rate of $1 \mathrm{MHz}$. A square with a length $a=500 \mu \mathrm{m}$ serves as example geometry. The target depth $d_{t}$ was set to $200 \mu \mathrm{m}$.

\section{A theoretical view of ultrashort pulse milling}

To clarify how process durations for milling applications result, a theoretical view is presented. Additionally, typical quality requirements for milling geometries are defined. With $(V / Q)$ as the ablated volume per energy, the ablated depth per scan $d_{\text {layer }}$ for a square is given by

$$
d_{\text {layer }}=\frac{f_{R} \cdot E_{p} \cdot(V / Q)}{d_{h} v_{s}}
$$

with the repetition rate $f_{R}$, the pulse energy $E_{p}$ and the scanning speed $v_{s}$. Consequently, the required number of scans to reach the target depth $d_{t}$ is $N=d_{t} / d_{\text {layer. }}$. The time required to ablated a layer is determined by the duration of a scan line $\tau_{\text {line }}=a / v_{s}$, the number of lines per ablated layer $a / d_{h}$ and the jump and acceleration times of each line $\tau_{\text {off. }}$ This leads to the equation

$$
\tau_{\text {layer }}=\frac{a^{2}}{d_{h} v_{s}}+\frac{a}{d_{h}} \cdot \tau_{\text {off }} .
$$

Finally, the total process duration $\tau_{\text {total }}$ results in

$$
\begin{aligned}
\tau_{\text {total }} & =N \cdot \tau_{\text {layer }}=\frac{d_{t}}{d_{\text {layer }}} \tau_{\text {layer }} \\
& =\frac{d_{t} v_{s} a}{f_{R} \cdot E_{p} \cdot(V / Q)} \cdot\left[\frac{a}{v_{s}}+\tau_{\text {off }}\right]
\end{aligned}
$$

This equation provides a very simple but often quite accurate estimate of the process duration. Additionally, it is now easy to see that the process duration increases linearly with the scan speed for constant $\tau_{\text {off }} . \tau_{\text {off }}$ corresponds to the time where the average laser power is unused. It accounts for a higher proportion of the total process duration if the edge length decreases or the scanning speed increases. This becomes particularly clear if the laser on time is set in relation to the process duration as follows:

$$
\frac{\tau_{\text {on }}}{\tau_{\text {total }}}=\left(N \cdot \frac{a^{2}}{d_{h} v_{S}}\right) \cdot\left(N \cdot \tau_{\text {layer }}\right)^{-1}=\frac{a}{a+v_{S} \cdot \tau_{\text {off }}}
$$

Equation 3 and 4 are plotted for $\tau_{\text {off }}=1 \mathrm{~ms}$ (typical value for galvanometer scanner with constant acceleration time) and $(V / Q)=1.7 \mathrm{~mm}^{3} / \mathrm{kJ}$ in Figure 1 . This illustrates that especially for small geometries, which often require the precision of ultrashort pulse milling; high scanning speeds hinder the exploitation of a high average power. For scanners without constant acceleration time there is even an optimal scanning speed [10]. However, if this optimal speed is below the material-specific critical scanning speed, it cannot be used

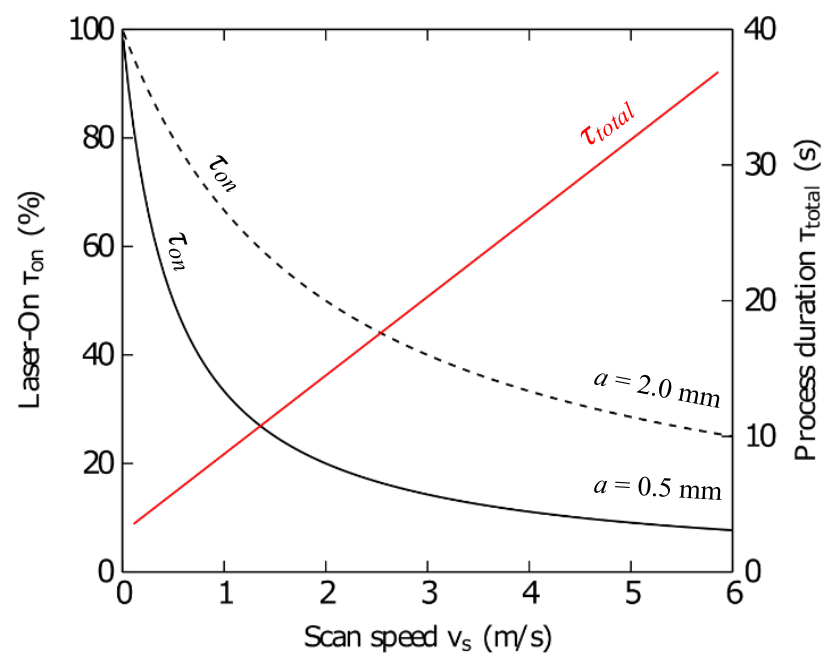

Fig. 1 Laser on time according to equation 4 for two edge lengths $a$ (left axis) and the total process duration according to equation 3 in red (right axis). The average power used decreases as the scanning speed increases (black lines).

for milling while maintaining the same surface quality. Therefore, it would be a great advantage to find a way to use low scanning speeds.

In addition to the process time, the machining quality is of course also of great importance in applications. A common type of requirement concerns the surface quality of the ground. Furthermore, high quality steep walls are often required. Therefore, we want to make the following requirements to our target geometry:

1. A smooth surface of the ground

2. Steep walls

3. Minimal damage at the edges and no edge deepening

Edge deepening is typically caused by reflected energy to the bottom of the structure, which can lead to an increase of absorbed fluence at the edges.

\subsection{The dual process strategy}

The aim of the dual process strategy is to suppress the formation of bumps by the repeated insertion of polishing steps. This allows the use of a higher ablation rate at a scanning speed below the critical speed $v_{\text {crit. }}$. For the conventional milling process, keeping the critical scanning speed limits the process duration according to equation 3 . Therefore, the dual process strategy effectively results in a higher ablation rate if the polishing steps take less time than the time saved by applying a lower scanning speed $\left(v_{s}<v_{\text {crit }}\right)$. The polishing effect is achieved by using $\mathrm{GHz}$ bursts with single pulse fluences below the ablation threshold [11]. In addition, the polishing steps are a key factor in avoiding edge deepening.

\section{Experiments}

\subsection{Milling with different scanning speeds}

As a first step, different scanning speeds in unidirectional scanning were investigated to determine the critical scan speed and the number of scans required to reach the target depth. Additionally, this first experiment provides guidance in selecting a scanning speed for the dual process strategy. Since the occurrence of the bumps correlates with the heat 


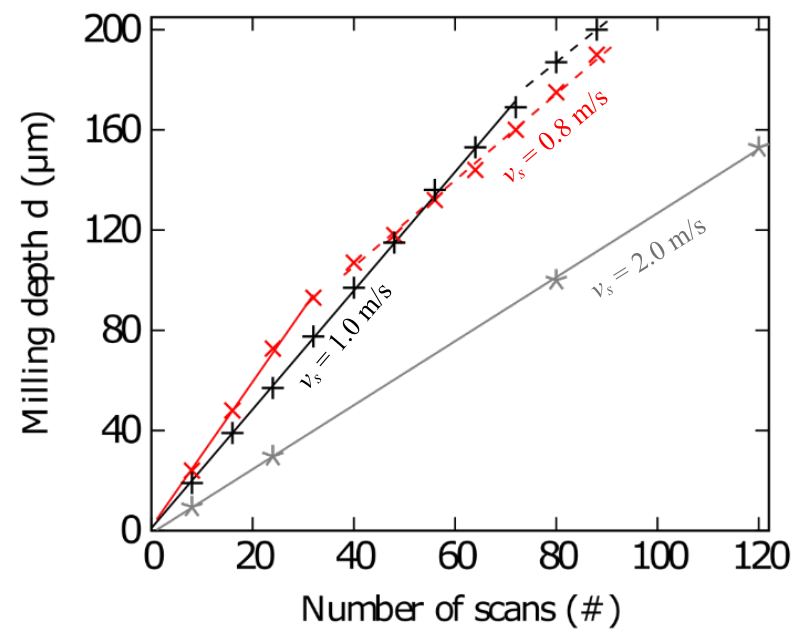

Fig 2 Milling depth at the center of the square against number of scans for different scanning speeds. The dashed part shows the ablation rate in the case of a bumpy surface, which is approximately $30-40 \%$ lower than for a smooth surface.

accumulation, they form earlier at lower scanning speeds. Therefore, it is advantageous to choose a speed that is below the critical speed but at which the bumps do not occur after only a few scans. Otherwise, more polishing steps must be inserted to maintain an even surface with a high ablation rate, as the occurrence of bumps significantly reduces the ablation rate, which is shown in Figure 2 for different scanning speeds. Consequently, the process duration of the dual process strategy follows approximately the equation

$$
\tau_{\text {dual }}=\widetilde{N} \cdot\left(n_{\text {abl }} \tau_{\text {layer }}+n_{\text {pol }} \tau_{\text {pol }}\right)
$$

with $n_{\mathrm{abl}}$ and $n_{\mathrm{pol}}$ as number of ablation and polishing steps, $\widetilde{N}$ as number of iterations and $\tau_{\text {layer }}$ and $\tau_{\text {pol }}$ as durations for one ablation and one polishing step, respectively. Equation 2 can also be used to calculate the time for a polishing step. Figure 3 shows that the occurrence of bumps is not a sudden phenomenon, instead they grow with the number of scans. We refer to bumps as soon as they have reached a size that leads to a significant reduction of the ablation rate. At the same moment, they appear dark under a light microscope and tend to oxidize strongly [1]. For the target depth of $200 \mu \mathrm{m}$, a scanning speed of $2.5 \mathrm{~m} / \mathrm{s}$ is sufficient to maintain a good surface quality and avoid strong bump formation.
The minimum number of scans required to reach the target depth is about 200, which corresponds to a process time of 20 seconds. If we set $v_{s}=1 \mathrm{~m} / \mathrm{s}$, the laser on time is doubled, as can easily be seen from equation 4 and Figure 1, and the process duration is reduced to 8.94 seconds. However, with $v_{s}=1 \mathrm{~m} / \mathrm{s}$, the first bumps occur at the edges of the geometry after 28 scans and increasingly spread over the entire surface, as shown in Figure 3. The reflections from the walls lead to a higher locally absorbed fluence, so that the bumps appear first at the edges. This local occurrence creates additionally a gradient in the ablation depth due to the worse ablation rate of the bumps, which is approximately $30 \%$ lower than in the case of a smooth surface. Since the bumps exhibit a lower ablation rate, using the polishing process as finishing is not very advantageous in terms of process time. Consequently, the use of the polishing process as an intermediate step to avoid bumps formation is more suitable.

\subsection{Milling using the dual process strategy}

In order to keep the bump formation at $v_{\mathrm{s}}=1 \mathrm{~m} / \mathrm{s}$ to a minimum, in this case a polishing step is inserted after every 28th ablation scan. For this purpose, the laser is switched into the $\mathrm{GHz}$ burst mode and the average power is divided into 256 sub-pulses. The switching between $\mathrm{GHz}$ mode and regular mode is currently done manually, as this is an experimental setup. These sub-pulses are sectioned into 8 bursts of 32 pulses with an intra-burst delay of $625 \mathrm{ps}$ and an extraburst repetition rate of $125 \mathrm{kHz}$. Between two burst there is an additional 20 ns burst pause (illustrated in Fig. 6). The single pulse peak fluence results consequently from

$$
F_{s p}=\frac{2 P_{a v}}{f_{R} N_{B} N_{p}} \cdot \frac{1}{\pi \omega_{0}^{2}}
$$

with $P_{a v}$ as average power, $f_{R}$ as repetition rate, $N_{B}$ as number of bursts and $N_{p}$ as number of pulses within a burst. A single pulse fluence of about $2 \mathrm{~mJ} / \mathrm{cm}^{2}$, which leads to a burst fluence of $F_{B}=N_{p} \cdot \mathrm{F}_{\mathrm{sp}}=64 \mathrm{~mJ} / \mathrm{cm}^{2}$ and a total fluence of $F_{\Sigma}=N_{B} \cdot F_{B}=512 \mathrm{~mJ} / \mathrm{cm}^{2}$ was used for polishing. A summary of the process parameter and the temporal energy distribution is shown in the appendix. By adding the polishing steps, the target depth is already reached after 84 scans of ablation and 6 scans of polishing. This number of ablation scans is 4 scans lower than the case without polishing, due to the absence of the bump-related reduction of the ablation
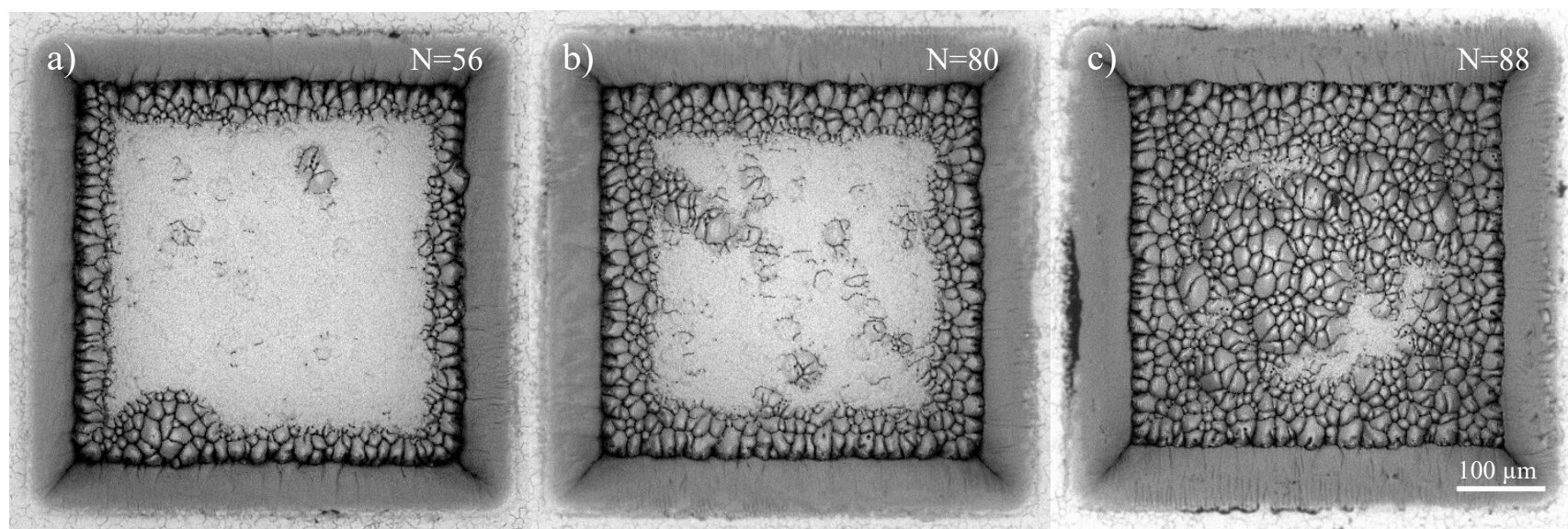

Fig. 3 Scanning electron image (SEM) of the milled square after different number of scans $N$. The bumps occur first at the edges. The used peak fluence is $F_{P}=0.73 \mathrm{~J} / \mathrm{cm}^{2}$ and the scan speed $1 \mathrm{~m} / \mathrm{s}$. 

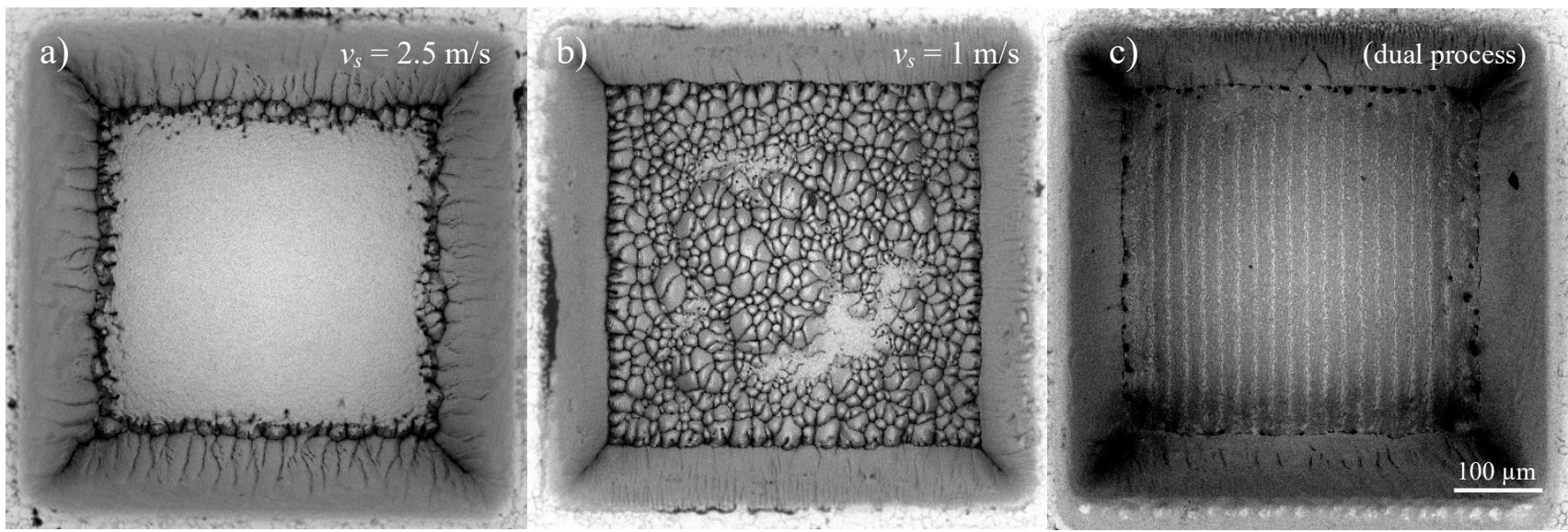

Fig. 4 SEM images of milled squares with a scan speed of a) $2.5 \mathrm{~m} / \mathrm{s}$ and b) $1 \mathrm{~m} / \mathrm{s}$ at a conventional process and $1 \mathrm{~m} / \mathrm{s}$ for c) using the dual process strategy. The surface roughness of the inner ground is $\mathrm{S}_{\mathrm{a}}=0.23 \mu \mathrm{m} \& \mathrm{~S}_{\mathrm{z}}=4.1 \mu \mathrm{m}$ (cutoff wavelength of $25 \mu \mathrm{m}$ ) for a), no longer measurable because of bumps for b) and $\mathrm{S}_{\mathrm{a}}=0.13 \mu \mathrm{m} \& \mathrm{~S}_{\mathrm{z}}=3.4 \mu \mathrm{m}$ for c). Although the scanning speed is the smallest, the edge and surface quality in c) is the best and just the line spacing during polishing limits the relatively high $\mathrm{S}_{\mathrm{z}}$ value.

rate. The total process time (including jump times) consists of $8.94 \mathrm{~s}$ for material removal and $0.44 \mathrm{~s}$ for polishing. Thus, the target depth is reached after a total process time of 9.38 seconds. Comparing this with the process time required with keeping the critical speed $(20 \mathrm{sec})$, the effective ablation rate is approximately doubled. In the following, the quality criteria will be checked using the examples.

Figure 4 shows milled squares at the target depth of $200 \mu \mathrm{m}$ using the dual process strategy and the cases of a scanning speed of 1 and $2.5 \mathrm{~m} / \mathrm{s}$ without polishing steps. Due to the discussed effect of wall reflections even at $v_{s}=2.5 \mathrm{~m} / \mathrm{s}$, bumps gradually occur at the edges. Regarding the quality requirements, neither of the two process results meets all of the criteria. The bumps at the edges are more clearly visible in the height profiles in Figure 5 for the case of $2 \mathrm{~m} / \mathrm{s}$. Figure 4 and 5 show also, that the reflections not only lead to bumps, which can be seen as an elevation in the height profile, but also to the unwanted edge deepening (Figure 5). The surface quality of the ground is sufficient for $v_{s}=2.5 \mathrm{~m} / \mathrm{s}$ but the best for the case of the dual process strategy. The remaining roughness or waviness is a result of the polishing process parameters. However, a further reduction could be achieved by using multiple scans at a lower power to the cost of some additional processing time.

Not only the best surface quality is achieved, when applying the dual process strategy but also the best wall and edge quality, as seen in the edge profiles and Figure 4c. The recurring polishing steps of the dual process strategy prevent the formation of the edge deepening. This is a great advantage compared to the conventional milling process, where such deepening normally occur along steep walls. Therefore, the dual process strategy also opens up advantages in terms of quality requirements. An overall summary of the results and applied process parameters is presented in table 1 and table 2 in the appendix A.

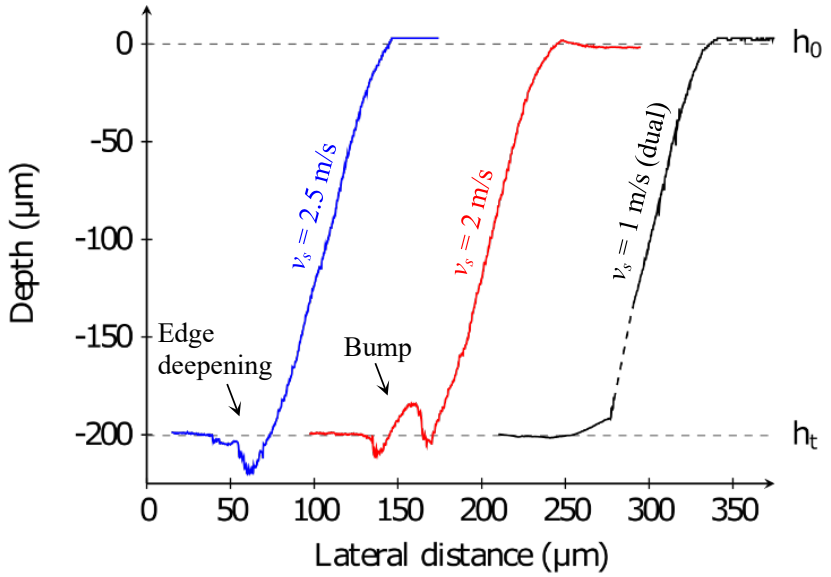

Fig. 5 Edge profiles of milled squares with different scan speeds. Profile a) with $v_{s}=2.5 \mathrm{~m} / \mathrm{s}$ in blue, b) with $v_{s}=2 \mathrm{~m} / \mathrm{s}$ in red and c) the dual process strategy with $v_{s}=1 \mathrm{~m} / \mathrm{s}$ in black. In the dashed part, interpolation is performed due to missing signal of the laser-scanning microscope. Even when the critical scanning speed is applied (blue line), reflections cause edge deepening. The edge steepness is about $75^{\circ}$ in all cases.

\section{Conclusion}

The process strategy of alternating laser milling and polishing steps ("dual strategy") leads to better surface quality and higher productivity of laser milling of metals. The recurring polishing steps of the strategy enable the use of scan speeds below the critical minimum scan speed, which increases the duty cycle significantly. It turns out that even the effect of edge deepening is suppressed, which is mostly observed for geometries with steep walls. Therefore, the dual process strategy proves to be an attractive approach to enhance the usable laser power of modern ultrashortpulsed lasers.

\section{Acknowledgments}

The author would like to thank Dr. A. Budnicki and C. Tan for their support with the modified TruMicro 2030 (Trumpf GmbH). 


\section{Appendix A}

Table 1 Summary of the results regarding process duration and quality requirements. Notation: ++ better, + good, - bad, -- worse

\begin{tabular}{lccc}
\hline \multicolumn{1}{c}{ Requirement } & Milling at $\boldsymbol{v}_{\boldsymbol{s}}=\boldsymbol{v}_{\mathbf{c r i t}} *$ & Milling at $\boldsymbol{v}_{\boldsymbol{s}}=\mathbf{1} \mathbf{~ m} \mathbf{s}$ & Dual process strategy \\
\hline Duration & $20 \mathrm{~s}$ & $8.94 \mathrm{~s}$ & $9.38 \mathrm{~s}^{* *}$ \\
Surface quality $\left(S_{a}, S_{z}\right)$ & $0.23 \mu \mathrm{m}, 4.1 \mu \mathrm{m}$ & bumpy / unsuitable & $0.13 \mu \mathrm{m}, 3.4 \mu \mathrm{m}$ \\
Edge deepening & -- & - & ++ \\
Steep walls $\left(>70^{\circ}\right)$ & + & + & +
\end{tabular}

*Minimum scan speed to avoid bumps **Process time without switching time

Table 2 Summary of the process parameter for milling and polishing

\begin{tabular}{lcc}
\hline \multicolumn{1}{c}{ Parameter } & Milling & Polishing \\
\hline Single pulse fluence & $0.73 \mathrm{~J} / \mathrm{cm}^{2}$ & $2 \mathrm{~mJ} / \mathrm{cm}^{2}$ \\
Repetition rate & $1 \mathrm{MHz}$ & $125 \mathrm{kHz}$ \\
Pulse duration & $1 \mathrm{p} \mathrm{s}$ & $1 \mathrm{ps}$ \\
Spot size & $42 \mu \mathrm{m}$ & $120 \mu \mathrm{m}$ \\
Line distance (hatch) & $7 \mu \mathrm{m}$ & $20 \mu \mathrm{m}$ \\
Scan speed & $1-2.5 \mathrm{~m} / \mathrm{s}$ & $0.25 \mathrm{~m} / \mathrm{s}$ \\
Hatch angle & $90^{\circ}$ & $90^{\circ}$
\end{tabular}

\section{Appendix B}

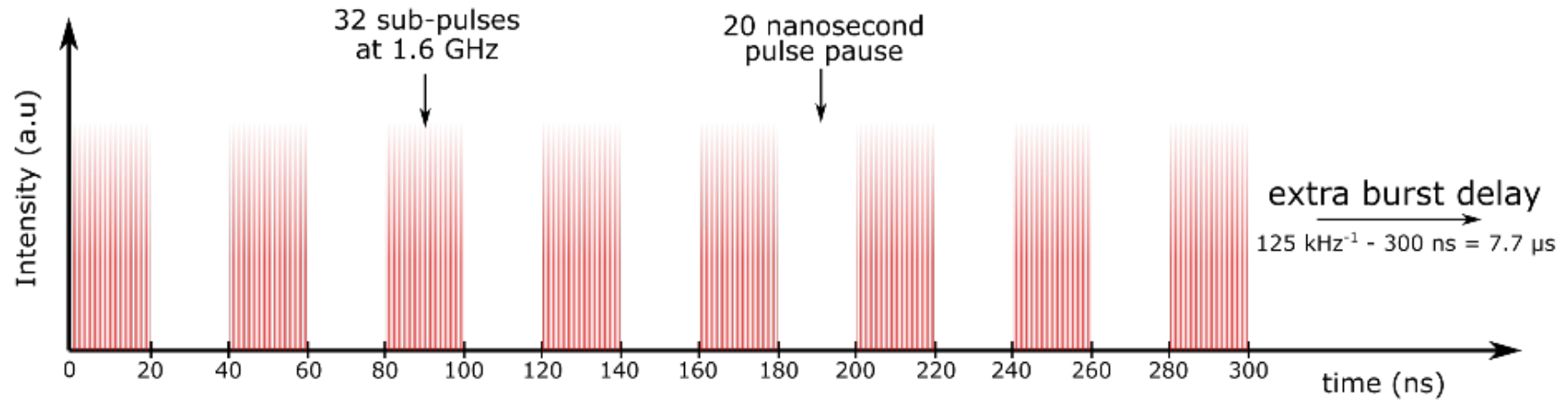

Fig. 6 Temporal structure of the burst for the polishing process. 256 pulses are divided into 8 burst packets, which are applied at $125 \mathrm{kHz}$ with an additional pulse pause of 20 nanoseconds.

\section{References}

[1] F. Bauer, A. Michalowski, T. Kiedrowski, and S. Nolte: Opt. Express, 23, (2015) 1035.

[2] R. Weber, T. Graf, P. Berger, V. Onuseit, M. Wiedenmann, C. Freitag, and A. Feuer: Opt. Express, 22, (2014) 11312.

[3] B. Jaeggi, S. Remund, R. Streubel, B. Goekce, S. Barcikowski, and B. Neuenschwander: J. Laser Micro/Nanoengin., 12, (2017).

[4] B. Neuenschwander, T. Kramer, B. Lauer, B. Jaeggi: Proc. SPIE, 9350, (2015) 93500U.

[5] Th. Kramer, Y. Zhang, St. Remund, B. Jaeggi, A. Michalowski, L. Grad, B. Neuenschwander: J. Laser Micro/Nanoengin., 12, (2017) 107.

[6] C. Kerse, H. Kalaycroglu, P. Elahi, B. Cetin, D. Kesim, Ö. Akçaalan, S. Yavas, M. Asik, B. Öktem, H. Hoogland, R. Holzwarth, and F. Ö. Ilday, Nature, 537, (2016) 84 .
[7] S. Hendow, H. Takahashi, M. Yamaguchi, and J. Xu: Proc. SPIE, 11268, (2020) 1126809.

[8] D.J. Förster, S. Faas, S. Gröninger, F. Bauer, A. Michalowski, R. Weber, and T. Graf, Appl. Surf. Sci. 440, (2018) 926.

[9] T. Hirsiger, M. Gafner, S. Remund, M. V. Chaja, A. Urniezius, S. Butkus, and B. Neuenschwander: Proc. SPIE, 11267, (2020) 112670T.

[10]B. Jaeggi, B. Neuenschwander, M. Zimmermann, M. Zecherle, and E. W. Boeckler: Proc. SPIE, 9735, (2016) 973513.

[11]F. Nyenhuis, A. Michalowski, and J. L'huillier: Proc. SPIE, 11268, (2020).

(Received: June 25, 2020, Accepted: October 19, 2020) 